\title{
Pattern of Antimicrobial Resistance to Escherichia Coli Among the Urinary Tract Infection Patients in Bangladesh
}

\author{
Goutam Kumar Acherjya ${ }^{1, ~ *, ~ K e y a ~ T a r a f d e r ~}{ }^{2}$, Rina Ghose ${ }^{3}$, Din Ul Islam², Mohammad Ali ${ }^{4}$, \\ Nazma Akhtar ${ }^{5}$, Rajashish Chakrabortty ${ }^{6}$, Satya Ranjan Sutradhar \\ ${ }^{1}$ Upazila Health Complex, Bagherpara, Jashore, Bangladesh \\ ${ }^{2}$ Department of Microbiology, Jashore Medical College \& Hospital, Jashore, Bangladesh \\ ${ }^{3}$ Department of Gynaecology and Obstretics, Jashore Medical College \& Hospital, Jashore, Bangladesh \\ ${ }^{4}$ Department of Hematology, National Institute of Cancer Research and Hospital, Dhaka, Bangladesh \\ ${ }^{5}$ Emergency Department, National Institute of Cardiovascular Disease, Dhaka, Bangladesh \\ ${ }^{6}$ Department of Medicine, Bangabandhu Sheikh Mujib Medical University (BSMMU), Dhaka, Bangladesh \\ ${ }^{7}$ Department of Medicine, Mymensingh Medical College \& Hospital, Mymensingh, Bangladesh
}

Email address:

gacherjya@hotmail.com(G. K. Acherjya)

*Corresponding author

To cite this article:

Goutam Kumar Acherjya, Keya Tarafder, Rina Ghose, Din Ul Islam, Mohammad Ali, Nazma Akhtar, Rajashish Chakrabortty, Satya Ranjan Sutradhar. Pattern of Antimicrobial Resistance to Escherichia Coli Among the Urinary Tract Infection Patients in Bangladesh. American Journal of Internal Medicine. Vol. 6, No. 5, 2018, pp. 132-137. doi: 10.11648/j.ajim.20180605.17

Received: July 21, 2018; Accepted: August 14, 2018; Published: October 10, 2018

\begin{abstract}
Urinary Tract Infection (UTI) is very common in our day-to-day clinical practice. Among all the organisms Escherichia coli (E coli) is the most common but antimicrobial resistance becomes an alarming issue for UTI management now a days. Aim of this study is to assess the pattern of antimicrobial resistance to $E$ coli among the UTI patients in Jashore, Bangladesh. This observational study was conducted from February, 2017 to January, 2018 in the district of Jashore, Bangladesh. We recruited 696 patients of both sex with UTI only infected by $E$ coli. We had excluded the patients with UTI caused by other than $E$ coli, female during menstruation, pregnancy, history of taking antibiotics within last 21 days, catheterization within 1 month and pelvic organ \& genito urinary tract surgery within 6 months. Evaluation of antimicrobial resistance was done according to the standard bacteriological methods. Mean age of our study cases was $41.46 \pm 17.21$ years with the range from 15 to 91 years. More than $85 \%$ participants were female with a sex ratio was $8: 1.5$. Reproductive age group ranged from 21 to 50 years was affected most commonly which constituted approximately two-third of our study. Our study revealed that the maximum antimicrobial resistance to $E$ coli was Cotrimoxazole (95.0\%), followed by Ceftazidime (75.7\%), Gentamicin (70.3\%), Amikacin (69.0\%), Imipenam (58.9 \%,), Cefixime (58.0\%), Ciprofloxacin (57.3\%), Azithromycin (56.0\%), Cefuroxime (46.6\%), Cefotaxime (37.4\%), Ceftriaxone (35.2\%), Meropenem (32.2\%), Nitrofurantion (4.7\%). With the high magnitude of antimicrobial resistance to $E$ coli among the UTI patients even with extended generation of Cephalosporins, Carbapenams, Ciprofloxacin, Cotrimoxazole, Azithromycin and Aminoglycosides, our recommendation as first line empirical treatment option in UTI should be Nitrofurantoin due to low resistance pattern.
\end{abstract}

Keywords: UTI, E coli, Antibiotic Resistance, Antibiotic Susceptibility

\section{Introduction}

Urinary tract infection (UTI) is one of the most common infectious diseases in the community practice with approximately 150 million people affected in the world each year accounting for nearly $25 \%$ of all infections. [1-2] Among all organisms $E$ coli causes $80-90 \%$ in uncomplicated UTIs. [3] A retrospective analysis using The Surveillance Network ${ }^{\circledR}$, USA in 2012 reported the most common pathogen isolated from female who visited U.S. outpatients in 2012 was E coli (64.9\%). [4] Urinary tract infection causes morbidities including 
pyelonephritis and cystitis which are resulted by presence of microorganisms in the urinary tract. [5] Though UTIs are related to minimum morbidity, the annual financial burden of the US alone costs at around 2 billion dollar. [6] However UTI is a common scenario in our daily clinical practice, but the increasing antimicrobial resistance is associated with treatment failure and overburden of healthcare cost around the globe. [7] Evidence shows that antimicrobial resistance pattern to urinary $E$ coli is growing gradually not only in the developing countries but also in the developed countries. In India from 2008 to 2013, the trend of antimicrobial resistance for E coli to third generation Cephalosporin, Fluoroquinolone and Carbapenams were increased from $70 \%$ to $83 \%, 78 \%$ to $85 \%$ and $10 \%$ to $13 \%$ respectively. [8] From 2000 to 2014, in German, Sweden, Spain and UK antimicrobial resistance against $E$ coli were increasing to ciprofloxacin from $2.2 \%$ to $20.2 \%, 0 \%$ to $7.3 \%, 14.7 \%$ to $30.8 \%$ and $0.5 \%$ to $15.3 \%$ respectively and to Trimethoprim from $22.5 \%$ to $36.8 \%, 8.8 \%$ to $16.9 \%, 25.1 \%$ to $37.3 \%$ and $14.9 \%$ to $46.0 \%$ respectively. In UK Nitrofurantoin resistance to urinary E. coli were increased from $0 \%$ to $6 \%$ in the same duration. [9] In USA from 2005 to 2009, increased $E$ coli resistance to Ampicillin from $39 \%$ to $43 \%$, Cefazolin from $4 \%$ to $7 \%$, Trimethoprime-Sulfamethoxazole $17 \%$ to $25 \%$, Fluoroquinolone from $7 \%$ to $16 \%$, Gentamicin from $3 \%$ to $7 \%$, and extended-spectrum Cephalosporins from 1\% to 3\%. [10] A time series analysis over five years in an Australian Tertiary Hospital reported significantly raised antimicrobial resistance in E coli against different antibiotics. [11] In the Study for Monitoring Antimicrobial Resistance Trends (SMART) in Canada and United States (US) confirmed increasing resistance in $E$. coli. after evaluation of resistance trend in $3498 \mathrm{E}$ coli induced UTI between 2010 to 2014 and found ExtendedSpectrum Beta-lactamase (ESBL) phenotype increasing (7.8$18.3 \%, \mathrm{P}<0.0001$ ) in US and susceptibility to Cephalosporins and Fluoroquinolones was significantly lower. But in Canada, no significant increasing trend $(10.4-13.0 \% . \mathrm{P}=0.079)$ of ESBL was found and lower than US rates. [12] Reports of US also confirmed increasing resistance in $E$. coli. by retrospective study using The Surveillance Network ${ }^{\circledR}$, USA in 2012 with a comparison with 2003 reports showing increasing resistance against Ciprofloxacin (3.6\% to $11.8 \%)$ and TrimethoprimSulfamethoxazole $(17.2 \%$ to $22.2 \%)$ with a lower resistance against Nitrofurantoin (from $0.7 \%$ to $0.9 \%$ ). [4]

As antimicrobial resistance is increasing all over the world even in the developed countries, so we have designed this study with the aim to see the effectiveness of different antimicrobials used against urinary tract infection with $E$ coli in terms of antimicrobial resistance in Jashore, Bangladesh.

\section{Methods}

\subsection{Study Design and Settings}

This observational study was conducted from February 2017 to January 2018 in the district of Jashore, Bangladesh. We recruited $694 \mathrm{E}$ coli positive UTI patients 15 years and above of both sex. Female patients during menstruation, pregnancy period, patients gave history of taking antibiotics within last 21 days, catheterization within 1 month, pelvic organ and genitourinary tract surgery within 6 month were excluded from the study. The purpose of the study was explained to each of the participants and verbal consent was taken from the participants regarding use of their urine culture report in our study. Institutional consent was approved from the superintendent of Jashore Medical College \& Hospital.

\subsection{Patients Profile}

Selected patients were both male and female of 15 years and above whom presenting to the physicians with the complaints of burning or painful micturition, increased frequency of micturition or lower abdominal pain.

\subsection{Urine Sampling}

Patients who had given informed consent to participate in the study been instructed verbally the collection of a clean catch mid-stream urine sample. Each participant was requested to give the early morning first voiding urine sample. After collecting the urine sample in a sterile container were processed for urine microscopy and culture in the same day in a renowned private pathological laboratory in Jashore, Bangladesh.

\subsection{Definition}

Microscopic examination revealed pus cell $>5 / \mathrm{HPF}$ in the centrifuged deposit of urine was considered as our study participants. The Microscope model CX23-LED. OLYMPUS (Japan) was used to identify pus cell in urine sample in our study.

\subsection{Bacterial Isolation and Identification}

The urine sample which revealed pus cell $>5 / \mathrm{HPF}$ in microscope were inoculated aseptically on Chromogenic agar (Hicrome UTI agar), Blood agar and MacConkey agar media and incubated 24 hours at $37^{\circ} \mathrm{C}$ aerobically. Completing the incubation period, we counted the number of bacteria per $\mathrm{ml}$ of urine. After passing overnight incubation period we had counted only the $E$ coli colony in the urine culture. The other bacterial colonies were excluded from our study.

\subsection{Antimicrobial Susceptibility Testing}

After identification of E coli in the urine sample we further demonstrated for antimicrobial susceptibility testing by Mueller-Hinton (MH) agar using Kirby-Bauer disc diffusion method against a panel of 13 antibiotics; Imipenum (10mcg), Ceftriaxone (30 mcg), Ciprofloxacin $(5 \mathrm{mcg})$, Cotromoxazole (25mcg), Ceftazidime (30 mcg), Cefotaxime (30 mcg), Gentamycin (10mcg), Nitrofurantoin (300 mcg), Azithromycin (15mcg), Cefixime $(5 \mathrm{mcg})$, Cefuroxime (30mcg), Meropenem (10mcg), Amikacin (30mcg). E coli ATCC 25922 were used as control strains for interpretations of antibiotics susceptibility testing. Antibiotics susceptibility 
was denoted as sensitivity and resistance by using the diameter of zone of inhibition as per the Clinical Laboratory Standard Institute (CLSI) guidelines. [13]

\subsection{Statistical Analysis}

All the data were analyzed by using Statistical Package for Social Sciences (SPSS) version 23. Categorical data was grouped as $\%$ and numbers and mean with standard deviation (SD) measured from continuous data.

\section{Results}

A total 696 eligible patients of both sex aged above 15 years whose urine culture grown $E$ coli were included in the our study. Out of total patients $102(14.7 \%)$ were male and $594(85.3 \%)$ patients were female gender. So, the majority patients in our study were female with a male and female sex ratio of 1.5:8 [Figure-1].



Figure 1. Sex distribution of the study population.

The mean age of our study population was $41.46 \pm 17.21$ with the age range from 15 to 91 years. The highest numbers of patients were from $2^{\text {nd }}$ to $5^{\text {th }}$ decades which constituted $62 \%$ of the total study population [Table 1 ].

Table 1. Age distribution of the study population.

\begin{tabular}{lll}
\hline Age in years & Number & Percentage (\%) \\
\hline$<20$ years & 74 & 10.6 \\
21-30 years & 178 & 25.6 \\
31-40 years & 109 & 15.7 \\
41-50 years & 144 & 20.7 \\
$51-60$ years & 79 & 11.4 \\
$61-70$ years & 77 & 11.1 \\
$>70$ years & 35 & 5.0 \\
Total & 696 & 100.0 \\
Mean \pm SD & $41.46( \pm 17.21)$ & Range 15-91 years \\
\hline
\end{tabular}

We had tested thirteen different commonly used antibiotics in urinary tract infection for evaluating the antibiotics susceptibility testing in our study. Among them Cotrimoxazole showed the highest number of antibiotics resistance to E coli which constituted $95 \%$ of the total patients. Nitrofurantoin constituted $4.7 \%$, the lowest rate of antibiotics resistance to $\mathrm{E}$ coli. The rest antibiotics in our study showed resistance to $E$ coli as follows, Ceftazidime (75.7\%), Gentamicin (70.3\%),
Amikacin (69.0\%), Imipenam (58.9\%,), Cefixime (58.0\%), Ciprofloxacin (57.3\%), Azithromycin (56.0\%), Cefuroxime (46.6\%), Cefotaxime (37.4\%), Ceftriaxone (35.2\%), Meropenem (32.2\%) [Table 2].

Table 2. Antibiotics resistant and sensitive pattern of the patients.

\begin{tabular}{lll}
\hline Name Antibiotics & Resistant & Sensitive \\
\hline Imipenum & $410(58.9 \% \%)$ & $286(41.1 \%)$ \\
Ceftriaxone & $245(35.2 \%)$ & $451(64.8 \%)$ \\
Ciproflaxacin & $399(57.3 \%)$ & $297(42.7 \%)$ \\
Cotrimoxazole & $661(95.0 \%)$ & $35(5.0 \%)$ \\
Ceftazidime & $527(75.7 \%)$ & $169(24.3 \%)$ \\
Cefotaxime & $260(37.4 \%)$ & $436(62.6 \%)$ \\
Gentamicin & $489(70.3 \%)$ & $207(29.7 \%)$ \\
Nitrofurantion & $33(4.7 \%)$ & $663(95.3 \%)$ \\
Azithromycin & $390(56.0 \%)$ & $306(44.0 \%)$ \\
Cefixime & $404(58.0 \%)$ & $292(42.0 \%)$ \\
Cefuroxime & $324(46.6 \%)$ & $372(53.4 \%)$ \\
Meropenem & $224(32.2 \%)$ & $472(67.8 \%)$ \\
Amikacin & $480(69.0 \%)$ & $216(31.0 \%)$ \\
\hline
\end{tabular}

\section{Discussion}

Urinary tract infections are second most common infections after respiratory tract infections among the human beings which may affects urethra, bladder or kidneys. [14] $E$ coli, a Gram negative bacillus which is responsible for more than $80 \%$ cases of urinary tract infections worldwide. [15] It is well postulated that UTI is more common among the woman due to the closer alignment of urethra to the anus and shorter urethra which we have found in our present study at a ratio of 8:1.5. [16-19]

Common risk factors causing UTIs are sexually active premenopausal women, use of spermicides for contraceptive, frequent change of sexual partners, age of the first UTI, maternal history of UTI, wearing tight undergarments, deferred voiding habit, pregnancy, DM and immunosuppression due to any cause. [20-22] So, reproductive age plays a vital role as a risk factor for developing UTI in both sex. In the meanwhile our study reveals that maximum age of UTI affected from $2^{\text {nd }}$ to $5^{\text {th }}$ decades which constitutes $62 \%$ of the total cohorts.

Antibiotics resistance to the bacterial infections can be a great barrier for the effective treatment options even in case of UTI. The antibiotics resistance is closely related with to greater mortality or morbidity and burden of total healthcare costs. Abuse of antibiotics, lack of patients education, unauthorized sale of antibiotics, limited access of health care systems, inadequate surveillance or regulatory systems, and non-human use of antimicrobial such as in animal production are main causative factors for resulting antibiotics resistance in the developing country. [23] Here we have used common form of antibiotics testing in our study to detect their resistance pattern to $E$ coli in UTI patients.

a. Cotrimoxazole: A few years back Cotrimoxazole was a commonly practicing antibiotic in many infections but its use becomes limited due to cutaneous side effects now a days. The present study shows that the highest antibiotics resistance to $E$ coli is Cotrimoxazole and which constitutes $95 \%$ of the total study population. 
One study supports our results where they have found Cotrimoxazole is one of the most resistant antibiotics to E coli. [24] Increasing resistance trends in US and Canada and lower susceptibility also support our finding. [4, 12, 25]

b. Nitrofurantoin: It is an oral form, cheap, less side effects and most effective antibiotics which are commonly used in UTIs patients in our country. We have found that Nitrofurantoin is only $4.7 \%$ resistance to $E$ coli in the present study and accounted the lowest resistance form antibiotics to it. So, Nitrofurantoin is still highly effective to E coli positive UTIs patients in Jessore Jashore, Bangladesh. Some studies favor and some goes against the result of 85 our present study. $[18,26,27]$ But less increasing resistance (from $0.7 \%$ to $0.9 \%)$ and higher susceptibility $(>80 \%)$ in US support our report. [4, 25]

c. Ciprofloxacin: The quinolone derivative, Ciprofloxacin is used empirically not only in UTIs but also in various forms infections due to its safety profile and cheap in price. Its oral form is widely used in uncomplicated UTI patients. Ciprofloxacin develops highly resistance to various infectious agents recently due to its inappropriate and misuse. This study has revealed $57.3 \%$ resistance to $E$ coli positive UTIs patients. This resistance rate of Ciprofloxacin to $E$ coli is higher than the other studies conducted in Bangladesh. Even Ciprofloxacin resistance rate $(79.66 \%)$ to $E$ coli is higher in one study in Saudi Arabia. [28-30] A multicenter study of 13 countries in the Asia-Pacific region from 2010-2013 reported lower susceptibility to Ciprofloxacin support our study. [31]

d. Azithromycin: Azithromycin is one of the macrolides which is highly popular for its dose convenient in use. Macrolides are used more often with some sexually transmitted disease (STD) caused urinary problems usually in combination with other antibiotics to eliminate severe UTIs. Unfortunately it shows $56 \%$ resistance to $E$ coli positive urine in these study populations. One study from West Bengal, India reported Azithromycin resistance to $E$ coli positive UTIs patients near about 30\%. [32]

e. Aminoglycosides: The most common Aminoglycosides are using in UTIs patients in our country are Gentamycin and Amikacin. Aminoglycosides are used usually in combination with other antibiotics to combat severe UTIs. Gentamycin and Amikacin are cheap, used parentally and less side effects except nephrotoxicity but this study reveals high resistance rate, $70.3 \%$ and $69.0 \%$ respectively to $E$ coli. A very recent study, the enzymatic resistance against Aminoglycosides to $E$ coli in northeastern Poland found that Gentamycin resistance rate was $59 \%$ and $11.4 \%$ in Amikacin. [33]

f. Cephalosporins: Second and third-generation Cephalosporins are commonly used empirical agents for both uncomplicated and complicated UTIs patients in any context. They are relatively expensive but safe even in case of pregnancy. Previously it was thought that they are highly susceptibility to UTIs patients but the present study shows highly resistance to $E$ coli eg: Cefuroxime (46.6\%), Cefixime (58.8\%), Ceftiaxone (35.2\%), Cefotaxime (37.4\%), and ceftazidime (75.7\%). A recent study reported that Cefuroxime $(72.41 \%)$ and Ceftriaxone $(66.58 \%)$ were higher resistance rate to urinary $E$ coli than that of the present study. [34] But one study conducted in the Ayatollah Rouhani Teaching Hospital of Babol Medical Sciences University in North of Iran reported the resistance rate of Ceftriaxone, Cefotaxime and Cefixime were $40.4 \%, 45.6 \%$ and $43.9 \%$ respectively which are near similar to our study. [24] But the $E$ coli exhibited higher resistance rate to Ceftazidime $(81.36 \%)$ and Cefotaxime $(76.27 \%)$ in a study of Saudi Arabia. [30]

g. Carbepenam: Imipenem and Meropenam of the Carbepenams group are the commonly agents using for the management of severe form of infections caused by extended -spectrum $\beta$ - lactamase (ESBL) positive $E$ coli in our country. Carbepenems are treated as a reserved drug to eliminate multi-drug resistance gram negative bacilli but unfortunately Meropenem and Imipenem are $32.2 \%$ and $58.9 \%$ resistance to $E$ coli positive UTI patients respectively in our study. Imipenem resistance rate to $E$ coli is $32.5 \%$ in a study of Pakistan. [35] Another study in Bangladesh has shown Meropnem resistance rate to $\mathrm{E}$ coli is $10.5 \%$. [36]

Antibiotics resistance depends on the genetic mutation and locality. So, geographical variation may be a determinant of difference magnitude of antimicrobial resistance pattern.

\section{Limitation of the Study}

Though the study includes significant number of cases but there are some potential limitations also. This includes-study conducted in a limited area, associated comorbidities were not evaluated, associated clinical presentations were not evaluated and distinctions of bacteriuria from UTI were not done.

\section{Conclusion}

High magnitude of antimicrobial resistance against Escherichia coli was observed among the UTI patients in Bangladesh even with extended generation of Cephalosporins, Carbapenems, Ciprofloxacin, Cotrimoxazole, Azithromycin and Aminoglycosides. Moreover, out of all other antibiotics Nitrofurantoin shown the least resistance against Escherichia coli. So, we recommended Nitrofurantoin as first line empirical treatment option in UTI patients.

\section{Authors' Disclosures of Potential Conflicts of Interest}

The author(s) indicated no potential conflicts of interest. 


\section{References}

[1] Keyhan H, Sedighi S, Mashayekhi B, Fathi M, Mokhtari M. Community Acquired Urinary Tract Infections' Etiological Organisms and Antibiotics Susceptibility Patterns. NephroUrology Monthly. 2017;9(5).

[2] Ahmed MA, Shukla GS, Bajaj HK. Incidence of urinary tract infections and determination of their susceptibility to antibiotics among pregnant women. J. Cell. Sci. Biotechnol. 2016:12-6.

[3] Zalmanovici Trestioreanu A, Green H, Paul M, Yaphe J, Leibovici L. Antimicrobial agents for treating uncomplicated urinary tract infection in women. Cochrane Database Syst Rev. 2010;10.

[4] Sanchez GV, Babiker A, Master RN, Luu T, Mathur A, Bordon J. Antibiotic resistance among urinary isolates from female outpatients in the United States in 2003 and 2012. Antimicrobial agents and chemotherapy. 2016 Feb 16:AAC-02897.

[5] Kulkarni R, Dhakal BK, Slechta ES, Kurtz Z, Mulvey MA, Thanassi DG. Roles of Putative Type II Secretion and Type IV Pilus Systems in the Virulence of Uropathogenic Escherichia coli. Beier D, ed. PLoS ONE. 2009; 4(3):e4752.

[6] Foxman B. The epidemiology of urinary tract infection. Nature Reviews Urology. 2010 Dec;7(12):653.

[7] Merz LR, Guth RM, Fraser VJ. Cost of Antimicrobial Resistance in Healthcare Settings: A Critical Review. InAntimicrobial Resistance 2010 (Vol. 6, pp. 102-119). Karger Publishers.

[8] Laxminarayan R, Chaudhury RR. Antibiotic Resistance in India: Drivers and Opportunities for Action. PLoS Medicine. 2016; 13(3):e1001974.

[9] Kahlmeter G, Åhman J, Matuschek E. Antimicrobial Resistance of Escherichia coli Causing Uncomplicated Urinary Tract Infections: A European Update for 2014 and Comparison with 2000 and 2008. Infectious Diseases and Therapy. 2015; 4(4):417-423.

[10] Swami SK, Liesinger JT, Shah N, Baddour LM, Banerjee R. Incidence of Antibiotic-Resistant Escherichia coli Bacteriuria According to Age and Location of Onset: A Population-Based Study From Olmsted County, Minnesota. Mayo Clinic Proceedings. 2012; 87(8):753-759.

[11] Fasugba O, Mitchell BG, Mnatzaganian G, Das A, Collignon P, Gardner A. Five-Year Antimicrobial Resistance Patterns of Urinary Escherichia coli at an Australian Tertiary Hospital: Time Series Analyses of Prevalence Data. Butaye P, ed. PLoS ONE. 2016; 11(10):e0164306.

[12] Lob SH, Nicolle LE, Hoban DJ, Kazmierczak KM, Badal RE, Sahm DF. Susceptibility patterns and ESBL rates of Escherichia coli from urinary tract infections in Canada and the United States, SMART 2010-2014. Diagnostic microbiology and infectious disease. 2016 Aug 1;85(4):459-65.

[13] Wayne PA. Clinical and laboratory standards institute. Performance standards for antimicrobial susceptibility testing.

[14] Mobley H, Alteri C. Development of a Vaccine against Escherichia coli Urinary Tract Infections. Pathogens. 2015; $5(1): 1$.
[15] Nicolle LE. Uncomplicated urinary tract infection in adults including uncomplicated pyelonephritis. Urologic Clinics of North America 2008; 35:1-12.

[16] O'Brien V, Hannan T, Schaeffer A, Hultgren S. Are you experienced? Understanding bladder innate immunity in the context of recurrent urinary tract infection. Current Opinion in Infectious Diseases. 2015; 28(1):97-105.

[17] Sharma G, Sharma S, Sharma P, Chandola D, Dang S, Gupta $\mathrm{S}$, et al. Escherichia coli biofilm:development and therapeutic strategies. J Appl Microbiol. 2016 Mar; 1-11.

[18] Abduzaimovic A, Aljicevic M, Rebic V, Vranic SM, Abduzaimovic K, Sestic S. Antibiotic Resistance in Urinary Isolates of Escherichia coli. Materia Socio-Medica. 2016; 28(6):416-419.

[19] Al-Badr A, Al-Shaikh G. Recurrent Urinary Tract Infections Management in Women: A review. Sultan Qaboos University Medical Journal. 2013; 13(3):359-367.

[20] Mohsin R, Siddiqui KM. Recurrent urinary tract infections in females. J Pak Med Assoc. 2010; 60:55-9.

[21] Scholes D, Hooton TM, Roberts PL, Stapleton AE, Gupta K, Stamm WE et al. Risk factors for recurrent urinary tract infection in young women. J Infect Dis. 2000; 182:1177-82.

[22] Franco VMA. Recurrent urinary tract infections. Best Pract \& Research Clinical Obstet Gynecol. 2005; 19:861-73.

[23] Ayukekbong JA, Ntemgwa M, Atabe AN. The threat of antimicrobial resistance in developing countries: causes and control strategies. Antimicrobial Resistance and Infection Control. 2017; 6:47.

[24] Shahandashti EF, Javanian M, Kouchaksaraei MM, Yeganeh B, Bijani A, Motevaseli E, et al. Resistance patterns of Escherichia coli causing urinary tract infection. Caspian Journal of Internal Medicine. 2015; 6(3):148-151.

[25] Jorgensen S, Zurayk M, Yeung S, Terry J, Dunn M, Nieberg P, Wong-Beringer A. Emergency department urinary antibiograms differ by specific patient groups. Journal of clinical microbiology. 2017 Jun 14:JCM-00481.

[26] Karlowsky JA, Thornsberry C, Jones ME, Sahm DF. Susceptibility of Antimicrobial-Resistant Urinary Escherichia coli Isolates to Fluoroquinolones and Nitrofurantoin, Clinical Infectious Diseases, 2003; 36(2):183-186.

[27] Zahra N, Rehman K, Aqeel R, Parveen A, Akash MSH. Assessment of urinary tract infection and their resistance to antibiotics in diabetic and non-diabetic patients. Bangabandu Sheikh Mujib Med Univ J, 2016; 9:151-155.

[28] Setu SK, Sattar A, Saleh AN, Roy CK, Ahmed M, Muhammadullah S, et al. Study of Bacterial pathogens in Urinary Tract Infection and their antibiotic resistance profile in a tertiary care hospital of Bangladesh, Bangladesh J Med Microbiol, 2016; 10 (01): 22-26.

[29] Akter T, Mia Z, Shahriar M. Antibiotic Sensitivity of Pathogens Causing Urinary Tract Infection, Bangladesh Pharmaceutical Journal, 2013; 16(1): 53-58.

[30] Alyamani EJ, Khiyami AM, Booq RY, Majrashi MA, Bahwerth FS, Rechkina E. The occurrence of ESBLproducing Escherichia coli carrying aminoglycoside resistance genes in urinary tract infections in Saudi Arabia. Annals of Clinical Microbiology and Antimicrobials. 2017; 16:1. 
[31] Jean SS, Coombs G, Ling T, Balaji V, Rodrigues C, Mikamo H, Kim MJ, Rajasekaram DG, Mendoza M, Tan TY, Kiratisin P. Epidemiology and antimicrobial susceptibility profiles of pathogens causing urinary tract infections in the Asia-Pacific region: Results from the Study for Monitoring Antimicrobial Resistance Trends (SMART), 2010-2013. International journal of antimicrobial agents. 2016 Apr 1;47(4):328-34.

[32] Saha S, Nayak S, Bhattacharyya I. Understanding the patterns of antibiotic susceptibility of bacteria causing urinary tract infection in West Bengal, India. Frontiers in Microbiology. 2014; 5:463.

[33] Ojdana D, Sieńko A, Sacha P, Majewski P, Wieczorek P, Wieczorek A, et al. Genetic basis of enzymatic resistance of E. coli to aminoglycosides. Adv Med Sci. 2018; 63(1):9-13.
[34] Kulkarni SR, Peerapur BV, Sailesh KS. Isolation and Antibiotic Susceptibility Pattern of Escherichia coli from Urinary Tract Infections in a Tertiary Care Hospital of North Eastern Karnataka. J Nat Sci Biol Med. 2017; 8(2): 176-180.

[35] Jafri SA, Qasim M, Masoud MS, Rahman M-, Izhar M, Kazmi S. Antibiotic resistance of E. coli isolates from urine samples of Urinary Tract Infection (UTI) patients in Pakistan. Bioinformation. 2014; 10(7):419-422.

[36] Akhtar N, Rahman R, Sultana S. Antimicrobial Sensitivity Pattern of Escherichia coli Causing Urinary Tract Infection in Bangladeshi Patients. American Journal of Microbiological Research, 2016; 4(4):122-125. 